\title{
First-Dose Coronavirus 2019 Vaccination Coverage among the Residents of Long-Term Care Facilities in France
}

\author{
Joël Belmin ${ }^{a}$ b Pierre Lutzler ${ }^{c}$ Patrick Hidoux ${ }^{d}$ Olivier Drunat ${ }^{\mathrm{e}}$ \\ Carmelo Lafuente-Lafuente ${ }^{a, b}, f$ the Investigators of the DU MedCo Network \\ aHôpital Charles Foix, Assistance Publique-Hôpitaux de Paris, Ivry-sur-Seine, France; b Faculté de médecine, \\ Sorbonne Université, Paris, France; 'EHPAD Saint Domnin, Digne les Bains, France; 'EHPAD L'Edelweiss, Gap, France; \\ eHôpital Bretonneau, Assistance Publique-Hôpitaux de Paris, Paris, France; 'CEpiA EA 7376 (Clinical Epidemiology \\ and Ageing Unit), Créteil, France
}

\section{Keywords}

Coronavirus-19 - Vaccine coverage $\cdot$ Long-term care facility

\section{Abstract}

Background: Long-term care facilities (LTCFs) experienced severe burden from the Coronavirus 2019 (COVID-19), and vaccination against SARS-CoV-2 is a major issue for their residents. Objective: The objective of this study was to estimate the vaccination coverage rate among the residents of French LTCFs. Method: Participants and settings: 53 medical coordinators surveyed 73 LTCFs during the first-dose vaccination campaign using the BNT162 b2 vaccine, conducted by health authorities in January and early February 2021. Measurements: in all the residents being in the LTCF at the beginning of the campaign, investigators recorded age, sex, history of clinical or asymptomatic COVID-19, serology for SARS-CoV-2 or severe allergy, current end-of-life situation, infectious or acute disease, refusal of vaccination by the resident or by the representative person of vaccine, and the final status, vaccinated or not. Results: Among the 4,808 residents, the average coverage rate for COVID-19 vaccination was $69 \%$, and
$46 \%$ of the LTCFs had a coverage rate $<70 \%$. Among unvaccinated residents, we observed more frequently a history of COVID-19 or a positive serology for SARS-CoV-2 (44.6 vs. $11.2 \%$ among vaccinated residents, $p<0.001$ ), a history of severe allergy ( 3.7 vs. $0.1 \%, p<0.001)$, end-of-life situation (4.9 vs. $0.3 \%, p<0.001$ ), current infectious or acute illness (19.6 vs. $0.3 \%, p<0.001$ ), and refusal of vaccination by resi-

List of the Investigators of the DU MedCo network: Drs. Folla Amara, Nadia Ammar, Eric Auboire, Ségolène Barneoud, Joëlle Batot, Sylvia Blazkova, Catherine Burugorri-Pierre, Hélène Cabrol, Mathieu Carbonell, Jessica Carranza, Aude Chartier, Dominique Chupin, Mirella Constans, Joëlle Da Col, Michel Davy, Emmanuelle De Gentile Pasquier, André Denjean, Olivier Drunat, Lucie Gagneur, Ewenn Gallou, Jean-Charles Grasset, Zohra Hassani, Patrick Hidoux, Sarra Jendoubi, Avidan Kogel, Sophie Kubas, Chahrazed Ladjal, Amina Lahlou, Guillaume Lalande, Florence Latour, Daniel Le Corre, Thierry Lebrun, Marc Loubière, Pierre Lutzler, Alexandra Meneses, Ghislaine Monier, Sauphia M'Touguy, Irina Neata, Sarra Nouri, Séverine Postaire, Didier Robert, Mihaela Rusinaru, Zedjiga Saidj-Lamari, Laurence Saudeau, Pascale Schmid-Gallean, Katia Sitbon, Emmanuel Soumah, Valérie Steff, Amélie Trarieux, Nathalie Tricot, Alphonse Tshiakatumba, Nicolas Valdenaire, Fanja Vergeade, and François Viatgé.
Correspondence to:

Joël Belmin, j.belmin@aphp.fr 
dents or representative persons (38.9 vs. $0.4 \%, p<0.001$ ). Conclusions: About 3 out of 10 residents remained unvaccinated, and half of the LTCFs had a coverage rate $<70 \%$. This suggests that COVID-19 will remain a threat to many LTCFs after the vaccination campaigns.

(c) 2021 S. Karger AG, Basel

\section{Introduction}

The Coronavirus 2019 (COVID-19) is responsible for a high burden in long-term care facilities (LTCFs) with a high incidence among residents and staff and high mortality among residents [1]. In a survey conducted in European countries [2], 40-60\% of all deaths related to COVID-19 were reported among LTCF residents, and in the USA, approximately 120,000 deaths related to COVID-19 were documented among LTCF residents on February 7 , 2021 [3]. A large body of literature has shown that LTCF residents are at risk of occurrence of respiratory infections, and that outbreaks are favored by the close-living conditions of LTCF residents and staff [4]. Advanced age, immunosenescence, multiple chronic illnesses, frailty, and disability are factors that explain why the fatality rate of COVID-19 is very high among LTCF residents $[5,6]$.

In this context, the health authorities in several countries, including the USA [7], the United Kingdom [8], and France [9], have placed the vaccination of LTCF residents among the top priorities of the COVID-19 vaccination campaigns, and immediately after the BNT162b2 vaccine (Pfizer-BionTech) was licensed for use in France, a vaccination campaign was implemented there for the LTCFs. Here, we present a first survey to estimate the vaccination coverage against COVID-19 among the residents of the French LTCFs.

\section{Materials and Methods}

French health authorities launched a vaccination campaign in the LTCFs shortly after the vaccine was licensed in France. In each region of France, the Agence Régionale de Santé (ARS) representing the Ministry of Health was responsible for providing doses of BNT162b2 vaccine in the LTCFs for residents eligible for vaccination. In addition, this vaccine was offered to healthcare professionals (HCPs) over 50 years of age who worked in the facility as employees or external HCPs and also to those under 50 years of age suffering from obesity or chronic disease. In practice, the medical coordinators and directors of each facility provided the ARS with a list of residents and caregivers who were eligible and willing to be vaccinated. For each facility, the vaccination date was planned by the ARS.

COVID-19 Vaccination Coverage in French Long-Term Care Facilities
For this survey, we invited LTCF medical coordinators who were students, former students, or teachers of the Sorbonne Université course that prepares physicians to work as LTCF medical coordinators (the Diplôme d'Université Médecin Coordonnateur en EHPAD). The medical coordinators were contacted by e-mail, and 60 of them volunteered to participate in the survey. They were asked to record for each resident present in their LTCF at the beginning of the vaccination campaign, age, sex, a history of COVID-19 (none, clinical, or asymptomatic), a history of SARSCoV-2 serology (none, negative, or positive), history of severe allergy of anaphylaxis type (yes/no), an end-of-life situation (yes/ no), a context of the current infectious or acute disease (yes/no), the expression of refusal of vaccination by the resident (yes/no) or by a representative of the resident (trusted person or legal representative) (yes/no), and finally whether the vaccine was prescribed (yes/no) and administered (yes/no). We also asked medical coordinators to record information about vaccination of the HCPs during this campaign, particularly the number of HCPs in the facility and the number of HCPs who received the first-dose vaccine. The data collection was closed on February 7, 2021.

\section{Statistics}

The independent variables recorded were compared between residents unvaccinated and vaccinated using the $t$ test and $\chi^{2}$ test. Analysis was done using STATA 16 software (StatCorp, USA). A $p<0.05$ was the level of significance.

\section{Results}

Fifty-three medical coordinators completed the survey and provided data about 73 LTCFs located in 10 French regions. The LTCF legal status was public for 32 facilities, private-for-profit for 23 , and private nonprofit for 18 . Seven medical coordinators who volunteered to participate in the study withdrew, 2 because they contracted COVID-19 and 5 due to lack of time for data collection. The first-dose campaign occurred from January 4 to February 7, 2021. Among the 4,808 residents being in the LTCFs at the beginning of the campaign, there were 3,608 females aged $88.3 \pm 8.3$ years and 1,200 males aged $83.3 \pm 10.6$ years $(p$ $<0.001)$. The distribution of the LTCFs according to the percentage of residents vaccinated is shown in Figure 1. The average coverage rate among the residents was 0.69 , and median was 0.72 (interquartile range $0.58-0.85$ ). There were $45.2 \%$ of the LTCFs with a coverage rate $<70 \%$.

The characteristics of the residents according to the vaccination status are shown in Table 1 . Unvaccinated status was significantly associated with a history of clinical or asymptomatic COVID-19, with a history of positive serology for SARS-CoV-2, with a history of severe allergy, endof-life context, and a current infectious or acute disease. In addition, refusal of vaccination was expressed by 349 residents (7.3\%) and by 292 representative persons (6.1\%). 
Fig. 1. Distribution of the long-term care facilities according to the percentage of residents vaccinated by a class of $10 \%$.

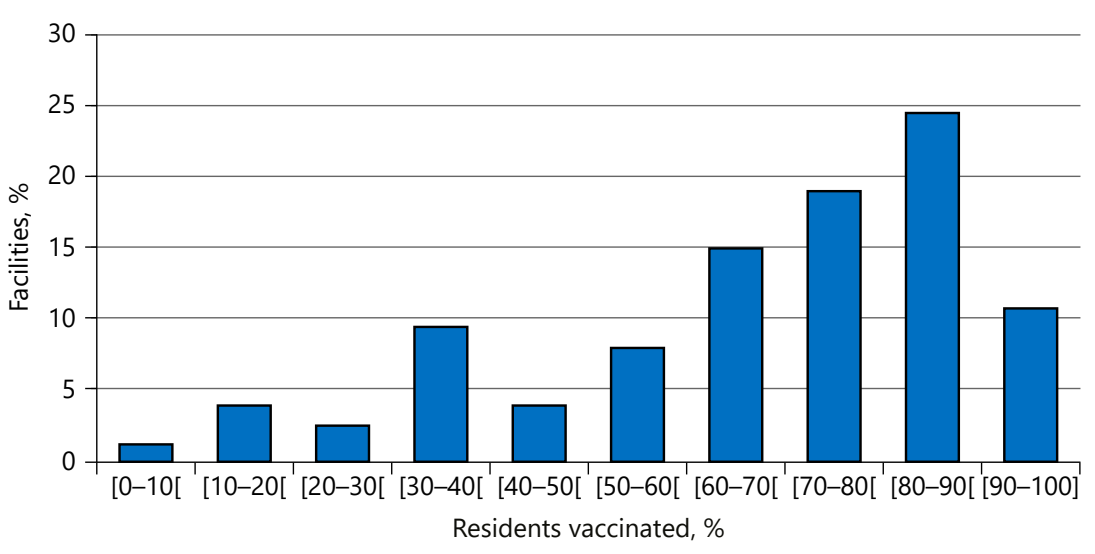

Table 1. Comparison of the characteristics of the 3,367 residents vaccinated against the COVID-19 and the 1,441 unvaccinated residents

\begin{tabular}{|c|c|c|c|c|}
\hline Age (mean and SD) & $87.0(9.6)$ & $86.6(9.9)$ & $87.2(9.6)$ & 0.065 \\
\hline \multicolumn{5}{|l|}{ Sex } \\
\hline Males & $1,200(24.9)$ & $362(25.1)$ & $838(24.9)$ & \multirow{2}{*}{0.884} \\
\hline Females & $3,608(75.1)$ & $1,079(74.9)$ & $2,529(75.1)$ & \\
\hline \multicolumn{5}{|l|}{ History of COVID-19 } \\
\hline Asymptomatic COVID-19 & $279(5.8)$ & $148(10.3)$ & $131(3.9)$ & \multirow[t]{2}{*}{$<0.001$} \\
\hline None & $3,793(78.9)$ & $799(55.4)$ & $2,994(88.9)$ & \\
\hline \multicolumn{5}{|l|}{ Past serology for SARS-CoV-2 } \\
\hline Positive & $318(6.6)$ & $159(11.0)$ & $159(4.7)$ & \multirow{3}{*}{$<0.001$} \\
\hline Negative & $757(15.7)$ & $149(10.3)$ & $608(18.1)$ & \\
\hline Not done & $3,733(77.6)$ & $1,133(78.6)$ & $2,600(77.2)$ & \\
\hline Residents refusing vaccination & $349(7.3)$ & $343(23.8)$ & $6(0.2)$ & $<0.001$ \\
\hline Representative person refusing vaccination & $292(6.1)$ & $283(19.6)$ & $9(0.3)$ & $<0.001$ \\
\hline Refusal from residents or representative persons & $574(11.9)$ & $561(38.9)$ & $13(0.4)$ & $<0.001$ \\
\hline
\end{tabular}

Information about vaccination of HCPs was obtained in 70 LTCFs. Among the $3225 \mathrm{HCPs}$ who were employees of the LTCF, 951 (29.5\%) were vaccinated during the campaign.

\section{Discussion/Conclusion}

This study shows that the average coverage rate for the COVID-19 vaccine was quite high among the LTCF residents, as 7 out 10 received the first-dose vaccine. How- ever, a substantial proportion of LTCFs remained with a low coverage rate after the first-dose vaccination campaign, and only $29.5 \%$ of HCPs were vaccinated.

The coverage rate recorded in French LTCFs for the COVID-19 vaccination is lower than that observed in American care homes where $78 \%$ of residents and $38 \%$ of HCPs received the first dose of vaccine [10]. The optimal coverage rate for COVID-19 vaccination to reduce the risk of SARS-CoV-2 transmission in LTCFs is not known. In addition, estimates of the clinical efficacy of COVID-19 in LTCF residents are still lacking, and based on 
studies of the influenza vaccine in LTCFs [11, 12], it is very likely that the immune responses of LTCF residents to the COVID-19 vaccine are lower than in the younger people who participated in the BNT162b2 vaccine clinical trial [13].

Our study provides some information on the factors associated with nonvaccination status. Refusal of vaccination by residents or their representatives was recorded in $12 \%$ of cases, which is a fairly low value compared to the high level of hesitancy recorded in France for other vaccines [14] and also to the high hesitancy for the future COVID-19 vaccines expressed by a representative sample of the French population in April 2020 [15]. Current acute illness, end-of-life situation, and allergy were unfrequently encountered factors associated with nonvaccination status. Direct or indirect signs of previous COVID-19 infection were significantly associated with nonvaccination status. Some physicians responsible for prescribing the vaccines probably considered the risk of recurrent COVID-19 infection to be low and thus did not vaccinate individuals with a history of COVID-19, although the French health authorities did not give specific guidelines on the relevance of vaccination for these individuals [9].

The main limitation of this study is that the LTCFs surveyed were not a representative sample of French LTCFs and that the recruitment of medical coordinators through a university course may have constituted a bias. In addition, the percentage of vaccine coverage among the HCPs in this study will be rapidly subject to changes and will probably increase in the next months when vaccine against SARS-CoV-2 will be proposed to HCPs younger than 50 years with no risk factors for severe COVID-19. We conclude that despite the SARS-CoV-2 vac- cination campaigns, the risk of new outbreaks of COVID-19 must still be considered in the LTCFs, and the fight against the transmission of the SARS-CoV-2 virus must remain active.

\section{Statement of Ethics}

This study was conducted ethically in accordance with the World Medical Association Declaration of Helsinki. It has been submitted and approved by the Comité d'Ethique de la Recherche of Sorbonne Universite (Paris, France) with the reference CER2021-010. Written consent was not required from the residents surveyed according to local laws regarding this type of survey. A comprehensive information package for residents, their proxies, and staff was carried out in each surveyed facility. These points were approved by the ethics committee.

\section{Conflict of Interest Statement}

The authors have no conflicts of interest to declare.

\section{Funding Sources}

The authors did not receive any funding.

\section{Author Contributions}

Conception and design of the study: J.B., P.L., P.H., O.D., and C.L.L. Involvement in protocol design adjustments, participant inclusion, and data recording: Investigators of the DU MedCo network. Drafting the manuscript: J.B., P.L., and C.L.L. Final approval of the manuscript: all the authors including the Investigators of the DU MedCo network.

\section{References}

1 Fallon A, Dukelow T, Kennelly SP, O’Neill D. COVID-19 in nursing homes. QJM. 2020; 113(6):391-2.

2 ECDC Public Health Emergency Team; Danis K, Fonteneau L, Georges S, Daniau C, BernardStoecklin S, et al. High impact of COVID-19 in long-term care facilities, suggestion for monitoring in the EU/EEA, May 2020. Euro Surveill Bull. 2020;25(22):2000956.

3 Centers for Medicare \& Medicaid Services. COVID-19 nursing home data. Baltimore, MD: US Department of Health and Human Services, Centers for Medicare \& Medicaid Services; 2020. Available from: https://data. cms.gov/stories/s/COVID-19-Nursing-
Home-Data/bkwz-xpvgexternalicon. Accessed 2021 Feb 7.

4 Lansbury LE, Brown CS, Nguyen-Van-Tam JS. Influenza in long-term care facilities. Influenza Other Respir Viruses. 2017;11(5):356-66.

5 Applegate WB, Ouslander JG. COVID-19 presents high risk to older persons. J Am Geriatr Soc. 2020;68(4):681.

6 D'Adamo H, Yoshikawa T, Ouslander JG. Coronavirus Disease 2019 in geriatrics and long-term care: the ABCDs of COVID-19. J Am Geriatr Soc. 2020;68(5):912-7.

7 Dooling K, McClung N, Chamberland M, McClung N, Chamberland M, Lee GM, et al. The advisory committee on immunization practices' interim recommendation for allocating initial supplies of COVID-19 vaccine - United States, 2020. MMWR Morb Mortal Wkly Rep. 2020;69:1857-9.

8 Department of Health and Social Care. Joint Committee on Vaccination and Immunisation: advice on priority groups for COVID-19 vaccination; 2020 Dec 30 [updated 2021 Jan 6]. Available from: https://www.gov.uk/government/publications/priority-groups-for-coronavirus-covid-19-vaccination-advice-fromthe-jcvi-30-december-2020/joint-committeeon-vaccination-and-immunisation-advice-onpriority-groups-for-covid-19-vaccination30-december-2020. Accessed 2021 Feb 7. 
9 Haute Autorité de Santé. Vaccins Covid-19: quelle stratégie de priorisation à l'initiation de la campagne?; 2020 Nov 30. Available from: https: //www.has-sante.fr/jcms/p_3221237/ $\mathrm{fr} /$ vaccins-covid-19-quelle-strategie-de-priorisation-a-l-initiation-de-la-campagne. Accessed 2021 Feb 7.

10 Gharpure R, Guo A, Bishnoi CK, Patel U, Gifford D, Tippins A, et al. Early COVID-19 first-dose vaccination coverage among residents and staff members of skilled nursing facilities participating in the pharmacy partnership for long-term care program - United States, December 2020-January 2021.
MMWR Morb Mortal Wkly Rep. 2021;70: $178-82$.

11 Fülöp T Jr, Wagner JR, Khalil A, Weber J, Trottier L, Payette H. Relationship between the response to influenza vaccination and the nutritional status in institutionalized elderly subjects. J Gerontol A Biol Sci Med Sci. 1999; 54(2):M59-64.

12 Muszkat M, Greenbaum E, Ben-Yehuda A, Oster M, Yeu'l E, Heimann S, et al. Local and systemic immune response in nursing-home elderly following intranasal or intramuscular immunization with inactivated influenza vaccine. Vaccine. 2003;21(11-12):1180-6.
13 Polack FP, Thomas SJ, Kitchin N, Absalon J, Gurtman A, Lockhart S, et al. Safety and efficacy of the BNT162b2 mRNA Covid-19 vaccine. N Engl J Med. 2020;383(27):260315.

14 Larson HJ, de Figueiredo A, Xiahong Z, Schulz WS, Verger P, Johnston IG, et al. The state of vaccine confidence 2016: global insights through a 67 -country survey. EBioMedicine. 2016;12:295-301.

15 COCONEL Group. A future vaccination campaign against COVID-19 at risk of vaccine hesitancy and politicisation. Lancet Infect Dis. 2020;20(7):769-70. 